\title{
Dosimetric comparison of different treatment modalities for stereotactic radiotherapy
}

\author{
Shih-Ming Hsu ${ }^{1,2,3^{*}}$, Yuan-Chun Lai ${ }^{1,4,5}$, Chien-Chung Jeng ${ }^{4}$ and Chia-Ying Tseng ${ }^{1,2}$
}

\begin{abstract}
Background: The modalities for performing stereotactic radiotherapy (SRT) on the brain include the cone-based linear accelerator (linac), the flattening filter-free (FFF) volumetric modulated arc therapy (VMAT) linac, and tomotherapy. In this study, the cone-based linac, FFF-VMAT linac, and tomotherapy modalities were evaluated by measuring the differences in doses delivered during brain SRT and experimentally assessing the accuracy of the output radiation doses through clinical measurements.
\end{abstract}

Methods: We employed a homemade acrylic dosimetry phantom representing the head, within which a thermoluminescent dosimeter (TLD) and radiochromic EBT3 film were installed. Using the conformity/gradient index (CGI) and Paddick methods, the quality of the doses delivered by the various SRT modalities was evaluated. The quality indicators included the uniformity, conformity, and gradient indices. TLDs and EBT3 films were used to experimentally assess the accuracy of the SRT dose output.

Results: The dose homogeneity indices of all the treatment modalities were lower than 1.25. The cone-based linac had the best conformity for all tumors, regardless of the tumor location and size, followed by the FFF-VMAT linac; tomography was the worst-performing treatment modality in this regard. The cone-based linac had the best gradient, regardless of the tumor location and size, whereas the FFF-VMAT linac had a better gradient than tomotherapy for a large tumor diameter $(28 \mathrm{~mm})$. The TLD and EBT3 measurements of the dose at the center of tumors indicated that the average difference between the measurements and the calculated dose was generally less than $4 \%$. When the $3 \%$ 3 -mm gamma passing rate metric was used, the average passing rates of all three treatment modalities exceeded $98 \%$.

Conclusions: Regarding the dose, the cone-based linac had the best conformity and steepest dose gradient for tumors of different sizes and distances from the brainstem. The results of this study suggest that SRT should be performed using the cone-based linac on tumors that require treatment plans with a steep dose gradient, even as the tumor is slightly irregular, we should also consider using a high dose gradient of the cone base to treat and protect the normal tissue. If normal tissues require special protection exist at positions that are superior or inferior to the tumor, we can consider using tomotherapy or Cone base with couch at $0^{\circ}$ for treatment.

Keywords: Linac, Stereotactic radiotherapy, Tomotherapy, Treatment planning systems, VMAT

\footnotetext{
* Correspondence: smhsu@ym.edu.tw

'Medical Physics and Radiation Measurements Laboratory, National

Yang-Ming University, Taipei, Taiwan, ROC

${ }^{2}$ Department of Biomedical Imaging and Radiological Sciences, National

Yang-Ming University, No. 155, Sec. 2, Li-Nong St., Beitou District, Taipei 112,

Taiwan, ROC

Full list of author information is available at the end of the article
} 


\section{Background}

Stereotactic radiosurgery (SRS) can be used to treat arteriovenous malformation, glioblastoma multiforme, and various metastasized tumors in the brain. Because lesions that are treated using SRS tend to be very small, and the method in which the doses are delivered differs from the traditional multi-fractionated dose-delivery mode, the required radiation in SRS is usually delivered in a single dose. In stereotactic radiotherapy (SRT), the required dose is fractionated into multiple doses. Because each of the doses in a single SRT or SRS treatment is extremely large, a high accuracy is necessary for the treatments, as well as a very steep dose gradient, to ensure the tumor is given a sufficiently high dose while the surrounding normal tissues are protected and left unharmed [1]. In recent years, advancements in linear accelerator (linac) based technologies, including developments in image-guidance systems, multileaf collimators (MLCs), and volumetric-modulated arc therapy (VMAT), have led to linac-based treatments that can achieve a high accuracy, steep gradients, and a high level of conformity [2-9].

Traditionally, the Gamma Knife has been the primary tool for performing brain SRS or SRT. However, the sites without this facility instead utilize tomotherapy and MLC or cone-based linac devices to perform SRT therapy. Tomotherapy has a linac mounted on a ring gantry, and a binary MLC is used to adjust the dose of the photon-beam irradiation in sync with the forward motion of the treatment couch, resulting in a helical and tomographic form of intensity modulated radiotherapy (TomoHelical IMRT). Linear accelerators may use either an MLC or a cone to shape and limit the field of radiation. Recent developments in flattening filter-free (FFF) high-dose models have led to a further reduction in the probability of patient movement, thus reducing the effects caused by patient movement. This has enabled the FFF-VMAT linac treatment modality to become a viable tool for performing SRT.

Different treatment modalities have different output dose characteristics, which may affect the radiation doses received by normal tissues surrounding the tumor [10-12]. Therefore, the main goal of this study is to compare the SRT treatment doses of the cone-based linac, FFF-VMAT linac, and tomotherapy treatment modalities and evaluate the differences between doses calculated according to treatment planning systems and measured radiation doses.

\section{Methods}

\section{Design of dosimetry phantom}

A homemade acrylic phantom of the head was created according to computed-tomography (CT) images of patients. EBT3 radiochromic films (Ashland, USA) and the cubic thermoluminescent dosimeters (Thermo,
USA) were installed within the coronal slices to measure the distribution of the radiation doses. In the region where the tumor was located the slices were $2 \mathrm{~mm}$ thick; the thickness of the remaining slices was $5 \mathrm{~mm}$, as shown in Fig. 1.

\section{Treatment planning}

CT (LightSpeed GE, USA) was applied to obtain images of the phantom, and the thickness of each CT slice was $1.25 \mathrm{~mm}$. The images were sent to a tomotherapy treatment planning system (TomoTherapy Planning Station Hi-ART $^{\oplus}$ version 4.3.2 Accuray, USA) and a Pinnacle treatment planning system (Pinnacle ${ }^{3 \odot}$ Version 9.8 Philips, USA). The TomoTherapy system designed all the planning related to tomotherapy, and the Pinnacle system designed all the planning for the cone-based and FFF-VMAT linacs. All necessary beam data were entered into the Pinnacle system, and commission tests were completed for this system. A structure with a diameter of $3 \mathrm{~cm}$ was placed on the CT images to simulate the location of the brainstem. To match the size of the collimating cone, spherical tumors 8,18 , and $28 \mathrm{~mm}$ in diameter were placed at distances of 1 and $6 \mathrm{~mm}$ from the borders of the brainstem, as shown in Fig. 2. The use of spherical tumors is expected to exclude the effects of tumor shapes in order to purely assess the differences in different modalities.

Different treatment plans were designed by the treatment planning systems according to the size of the tumors. To exclude the effects of the beam angle, all the cone-based linac and FFF-VMAT linac plans used the arc-therapy method and the same beam angles, as shown in Fig. 3. The beam angles were as follows: counterclockwise from $179^{\circ}$ to $345^{\circ}$ with a collimator angle of $0^{\circ}$ and a couch angle of $0^{\circ}$, counter-clockwise from $210^{\circ}$ to $180^{\circ}$ with a collimator angle of $10^{\circ}$ and a couch angle of $0^{\circ}$, counter-clockwise from $179^{\circ}$ to $0^{\circ}$ with a collimator angle of $330^{\circ}$ and a couch angle of $330^{\circ}$, counterclockwise from $179^{\circ}$ to $0^{\circ}$ with a collimator angle of $310^{\circ}$ and a couch angle of $300^{\circ}$, counter-clockwise from $179^{\circ}$ to $0^{\circ}$ with a collimator angle of $260^{\circ}$ and a couch angle of $270^{\circ}$, and clockwise from $180^{\circ}$ to $0^{\circ}$ with a collimator angle of $250^{\circ}$ and a couch angle of $60^{\circ}$.

The accelerator used for the cone-based linac was a 6MV Elekta Synergy (Sweden) photon. Cone diameters of 10, 20, and $30 \mathrm{~mm}$ were used. The accelerator used for the FFF-VMAT linac was a 6-MV FFF Elekta Axesse (Sweden) photon beam and was used in conjunction with a $5 \mathrm{~mm}$ wide Elekta Agility high-speed MLC. The following parameters were used for the TomoTherapy system (Accuray, USA): modulation factor of 3.0, field width of $1 \mathrm{~cm}$, pitch of 0.086 .

All treatment planning were planned by the same planner. In all treatment plans, a dose of 600 cGy was 

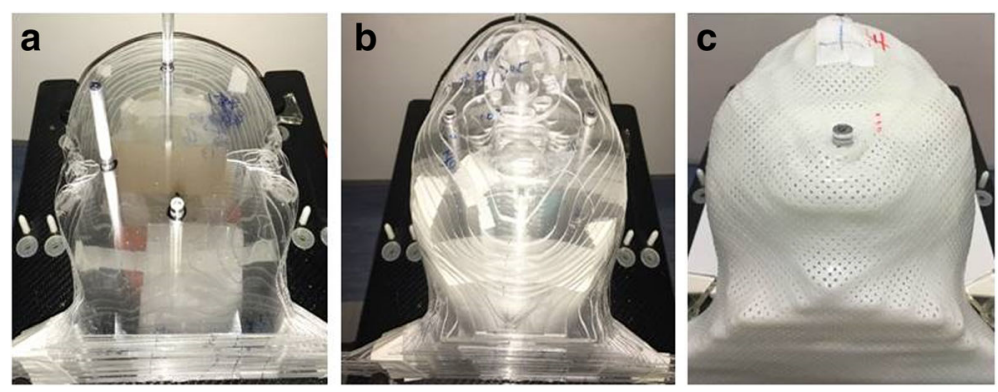

Fig. 1 Homemade acrylic dosimetry phantom of the head: (a) coronal slices and fixing rods, (b) head phantom formed from all of the coronal slices, and (c) head phantom fixed by a mask

delivered to the tumors, and the conditions were optimized to maintain $98 \%$ dose coverage on the tumor, with the maximum tumor dose not exceeding $125 \%$ to minimize the doses incident on the brainstem and normal brain tissue. Maximum dose constrain of brainstem for tumor away from brainstem $1 \mathrm{~mm}$ and $6 \mathrm{~mm}$ were limited to 500 cGy and 200 cGy, respectively and $D_{1 \%}$ of these were limited to $150 \mathrm{cGy}$ and $100 \mathrm{cGy}$, respectively. Auxiliary $\mathrm{ROI}_{\mathrm{s}}$ such as "Ring" structure were also used to decrease the dose of normal tissue during planning. The three-dimensional (3D) dose grid of the three axes of the Pinnacle system was set to $1 \mathrm{~mm}$ and that of the tomotherapy system was set to "fine".

\section{Analytic indicators of dose quality}

The homogeneity index (HI) [13], conformity/gradient index (CGI), and Paddick indices were used to compare the quality of the treatment plans. The HI was used to describe the homogeneity of the dose within the tumor. The HI is defined as

$$
\mathrm{HI}=\mathrm{MD} / \mathrm{PD},
$$

where MD is the maximum dose within the tumor, and PD is the $100 \%$ prescription dose. The HI of a perfect treatment plan is 1 ; if the $80 \%$ isodose curve is selected as the prescription dose, then the $\mathrm{HI}$ becomes 1.25 instead.

The CGI is a holistic index that consists of the conformity index $\left(\mathrm{CGI}_{\mathrm{c}}\right)$ and the gradient index $\left(\mathrm{CGI}_{\mathrm{g}}\right)$ [14] . The CGI is defined as

$$
C G I=\left(C G I_{C}+C G I_{g}\right) / 2
$$

The $\mathrm{CGI}_{\mathrm{c}}$ is used to describe the relationship between the volume of the tumor and the volume covered by the dose. The $\mathrm{CGI}_{\mathrm{c}}$ is defined as

$$
C G I_{C}=\left(\frac{T V}{P I V}\right) \times 100
$$

PIV is the volume covered by the $100 \%$ prescription dose curve, and TV is the volume of the tumor. $\mathrm{CGI}_{\mathrm{c}}=100$ corresponds to perfect conformity of the treatment planning. .

The $\mathrm{CGI}_{\mathrm{g}}$ is the effective difference in radius between the volumes covered by the $50 \%$ and $100 \%$ doses; it is used to evaluate the decrement of the dose in the highdose region (50\% and above) and is defined as follows:
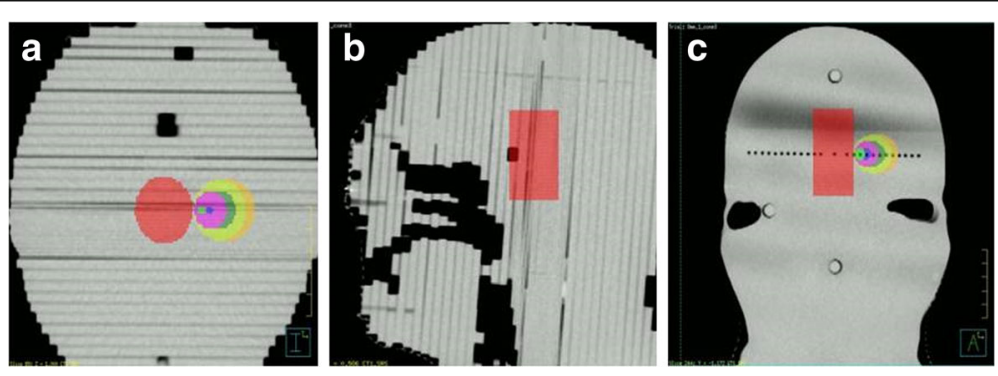

Fig. $2 \subset$ images of the homemade acrylic dosimetry phantom of the head: (a) transverse view, (b) sagittal view, (c) coronal view (Red, the simulated brainstem; Green, tumor of $8 \mathrm{~mm}$ diameter and $1 \mathrm{~mm}$ away from brainstem; Blue, tumor of $8 \mathrm{~mm}$ diameter and $6 \mathrm{~mm}$ away from brainstem; Pink, tumor of $18 \mathrm{~mm}$ diameter and $1 \mathrm{~mm}$ away from brainstem; Dark-green, tumor of $18 \mathrm{~mm}$ diameter and $6 \mathrm{~mm}$ away from brainstem; Yellow-green, tumor of $28 \mathrm{~mm}$ diameter and $1 \mathrm{~mm}$ away from brainstem; Orange, tumor of $28 \mathrm{~mm}$ diameter and $6 \mathrm{~mm}$ away from brainstem.) 


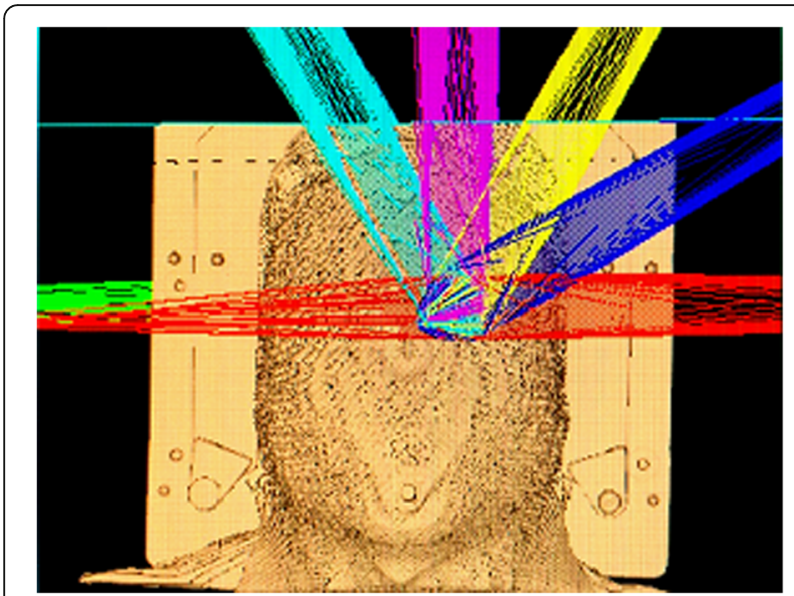

Fig. 3 All the beam angles used by the Pinnacle treatment planning system

$$
C G I_{g}=100-\left\{100 \times\left[\left(R_{e f f, 50 \% R x}-R_{e f f, R x}\right)-0.3 \mathrm{~cm}\right]\right\}
$$

$R_{e f f, R x}$ refers to the effective radius of the volume covered by the $100 \%$ prescription dose curve, and $R_{\text {eff, }} 50 \% R x$ is the effective radius of the volume covered by the $50 \%$ prescription dose curve, with $R_{\text {eff }}$ defined as

$$
R_{\text {eff }}=\sqrt[3]{\frac{3 V}{4 \pi}}
$$

$\mathrm{V}$ is the volume covered by the required dose. This $3 \mathrm{~mm}$ in distance between $R_{\text {eff, }} 50 \% R x$ and $R_{\text {eff, } R x}$ gradient was obtained empirically from clinical radiosurgery planning cases, and corresponds to the possible gradient with linac radiosurgery when using multiple noncoplanar arcs. As $\mathrm{CGI}_{\mathrm{g}}$ more than 100, it corresponds to less gradient than an optimum $3 \mathrm{~mm}$ empirically..

The Paddick indices are clinically used to describe the conformity [15] and gradient [16] of the treatment plan. The Paddick indices include the conformity index $\left(\mathrm{CI}_{\text {Paddick }}\right)$ and the gradient index $\left(\mathrm{GI}_{\text {Paddick }}\right)$. As the $\mathrm{CGI}_{\mathrm{c}}$ is unable to present the degree of tumor-volume coverage for a specified prescription dose curve, the $\mathrm{CI}_{\text {Paddick }}$ complements the $\mathrm{CGI}_{\mathrm{c}}$ by describing the volume covered by the prescription dose as well as the relationship between the tumor volume covered by the prescription dose and the overall volume of the tumor. The $\mathrm{CI}_{\text {Paddick }}$ is defined as

$$
\mathrm{CI}_{\text {Paddick }}=\frac{\left(T V_{P I V}\right)^{2}}{T V \times P I V}
$$

where PIV refers to the volume covered by the $100 \%$ prescription dose curve, TV is the tumor volume, and TVPIV is the tumor volume covered by PIV. This index represents the degree to which a tumor is covered by a specified isodose curve. For a perfect treatment plan, $\mathrm{CI}_{\text {Paddick }}=1$.

The $\mathrm{GI}_{\text {Paddick }}$ describes the decrement of the dose in the high-dose region (50\% and above) and is defined as

$$
\frac{G I_{\text {Paddick }}=V_{50 \%}}{V_{100}}
$$

where V $50 \%$ is the volume covered by $50 \%$ of the prescription dose, and $\mathrm{V} 100 \%$ is the volume covered by $100 \%$ of the prescription dose. The $\mathrm{GI}_{\text {Paddick }}$ can represent the degree to which normal tissues outside the tumor are protected. A perfect treatment plan must have a value of the $\mathrm{GI}_{\text {Paddick }}$ that approaches 1 .

\section{Radiation dose measurements}

In this work, cubic TLD-100 dosimeters and EBT3 films were used to measure the radiation dose [17-22], with the dose at each tumor location measured three times. Each cone-based linac and FFF-VMAT linac measurement was accompanied by alignment using the six degree-of-freedom image-guided cone-beam CT of the accelerator. MVCT was used to align the tomotherapy measurements to correct for 3D position shifts and rollangle deviations in the rotating gantry. FILM QATM Version 2.2 was used to evaluate the profile changes in the right-left $(\mathrm{R}-\mathrm{L})$ and superior-inferior (S-I) directions, and gamma evaluation was used to determine the differences between the calculated dose of the treatment plan and the two-dimensional planar dose measured by the EBT3 films. Because SRT treatments characteristically require a high level of positional accuracy and a steep gradient, the $3 \% 3-\mathrm{mm}$ and $5 \% 1-\mathrm{mm}$ criteria were chosen as the gamma passing rate metrics for assessing the differences in the planar doses.

\section{Statistical analysis}

The Mann-Whitney test (Statistical Package for the Social Sciences, IBM Corporation, New York, USA) was used to assess the statistical significance of the gamma analysis results for the different techniques.

\section{Results}

\section{Dose-quality analysis}

The results of the cone-based linac, FFF-VMAT linac, and tomotherapy dose-quality analyses are shown in Table 1 . The average HI values of the cone-based linac, the FFF-VMAT linac, and tomotherapy were $1.20 \pm 0.03$, $1.21 \pm 0.03$, and $1.23 \pm 0.02$, respectively. As it was strictly specified that the maximum dose on the tumors did not exceed the $125 \%$ of the prescription dose during the creation of the treatment plans, none of the average HIs of the treatment plans exceeded 1.25. The lowest HI 
Table $1 \mathrm{HI}, \mathrm{CGl}$, and Paddick indices calculated by the treatment planning system using the cone-based linac, FFF-VMAT linac, and tomotherapy treatment modalities

\begin{tabular}{|c|c|c|c|c|c|c|c|c|c|c|c|c|c|c|c|c|c|c|}
\hline \multirow{3}{*}{$\begin{array}{l}\text { Tumor } \\
\text { Diameter } \\
\text { Distance } \\
\text { from } \\
\text { brainstem } \\
\text { Modality }\end{array}$} & \multicolumn{6}{|l|}{$8 \mathrm{~mm}$} & \multicolumn{6}{|c|}{$18 \mathrm{~mm}$} & \multicolumn{6}{|c|}{$28 \mathrm{~mm}$} \\
\hline & \multicolumn{3}{|l|}{$1 \mathrm{~mm}$} & \multicolumn{3}{|l|}{$6 \mathrm{~mm}$} & \multicolumn{3}{|l|}{$1 \mathrm{~mm}$} & \multicolumn{3}{|l|}{$6 \mathrm{~mm}$} & \multicolumn{3}{|l|}{$1 \mathrm{~mm}$} & \multicolumn{3}{|l|}{$6 \mathrm{~mm}$} \\
\hline & $\begin{array}{l}\text { Cone- } \\
\text { based }\end{array}$ & $\begin{array}{l}\text { FFF- } \\
\text { VMAT }\end{array}$ & $\overline{\text { Tomo }}$ & $\begin{array}{l}\text { Cone- } \\
\text { based }\end{array}$ & $\begin{array}{l}\text { FFF- } \\
\text { VMAT }\end{array}$ & $\overline{\text { Tomo }}$ & $\begin{array}{l}\text { Cone- } \\
\text { based }\end{array}$ & $\begin{array}{l}\text { FFF- } \\
\text { VMAT }\end{array}$ & $\overline{\text { Tomo }}$ & $\begin{array}{l}\text { Cone- } \\
\text { based }\end{array}$ & $\begin{array}{l}\text { FFF- } \\
\text { VMAT }\end{array}$ & $\overline{\text { Tomo }}$ & $\begin{array}{l}\text { Cone- } \\
\text { based }\end{array}$ & $\begin{array}{l}\text { FFF- } \\
\text { VMAT }\end{array}$ & Tomo & $\begin{array}{l}\text { Cone- } \\
\text { based }\end{array}$ & $\begin{array}{l}\text { FFF- } \\
\text { VMAT }\end{array}$ & $\overline{\text { Tomo }}$ \\
\hline$\overline{\mathrm{HI}}$ & 1.24 & 1.25 & 1.20 & 1.23 & 1.25 & 1.23 & 1.20 & 1.19 & 1.25 & 1.20 & 1.17 & 1.24 & 1.17 & 1.23 & 1.21 & 1.16 & 1.19 & 1.23 \\
\hline $\mathrm{CGI}_{c}$ & 84.59 & 48.89 & 47.96 & 86.25 & 67.93 & 46.79 & 92.02 & 75.66 & 69.75 & 95.35 & 73.99 & 67.76 & 98.99 & 95.44 & 86.04 & 99.84 & 92.27 & 86.68 \\
\hline $\mathrm{CGI}_{\mathrm{g}}$ & 103.25 & 66.45 & 74.39 & 103.15 & 74.30 & 75.96 & 88.43 & 60.53 & 60.61 & 89.33 & 59.71 & 62.68 & 74.02 & 52.79 & 48.02 & 75.26 & 61.62 & 50.49 \\
\hline CGI & 93.92 & 57.67 & 61.18 & 94.71 & 71.11 & 61.38 & 90.23 & 68.10 & 65.18 & 92.34 & 66.85 & 62.22 & 86.50 & 74.11 & 67.03 & 87.55 & 76.95 & 68.58 \\
\hline Cl paddick & 0.82 & 0.48 & 0.47 & 0.84 & 0.66 & 0.45 & 0.90 & 0.73 & 0.68 & 0.92 & 0.72 & 0.66 & 0.94 & 0.92 & 0.84 & 0.95 & 0.88 & 0.81 \\
\hline Gl Paddick & 4.23 & 10.97 & 9.07 & 4.28 & 10.58 & 8.57 & 3.02 & 4.90 & 4.83 & 3.00 & 4.93 & 4.62 & 2.73 & 3.67 & 4.14 & 2.69 & 3.21 & 3.90 \\
\hline
\end{tabular}

(1.16) was observed when the cone-based linac was used to treat $28 \mathrm{~mm}$ diameter tumors that were located $6 \mathrm{~mm}$ from the brainstem.

The average CGI values for the cone-based linac, the FFF-VMAT linac, and tomotherapy were $90.88 \pm 3.37$, $69.13 \pm 6.74$, and $64.26 \pm 3.13$, respectively. In general, the FFF-VMAT linac had higher CGI values than tomotherapy except for tumors with small distance from the brainstem. The average $\mathrm{CGI}_{\mathrm{c}}$ values for the conebased linac, the FFF-VMAT linac, and tomotherapy were $92.84 \pm 6.41,75.70 \pm 17.01$, and $67.50 \pm 17.48$, respectively. For all distances from the brainstem and tumor diameters, the cone-based linac exhibited the highest $\mathrm{CGI}_{\mathrm{c}}$ values, followed by the FFF-VMAT linac, and finally tomotherapy.

The average $\mathrm{CGI}_{\mathrm{g}}$ values for the cone-based linac, the FFF-VMAT linac, and tomotherapy were $88.91 \pm 12.78,62.57 \pm 7.23$, and $62.03 \pm 11.65$, respectively. The FFF-VMAT linac had higher $\mathrm{CGI}_{\mathrm{g}}$ values than tomotherapy for large-diameter tumors $(28 \mathrm{~mm})$, with ratios of 52.79: 48.02 and 61.62: 50.49 for distances of 1 and $6 \mathrm{~mm}$ from the brainstem, respectively. The average $\mathrm{CI}_{\text {Paddick }}$ for the cone-based linac, the FFF-VMAT linac, and tomotherapy were $0.90 \pm 0.05,0.73 \pm 0.16$, and $0.65 \pm 0.16$, respectively. Regardless of the tumor diameter and distance from the brainstem, the cone-based linac exhibited the best $\mathrm{CI}_{\text {Paddick }}$ values, followed by the FFF-VMAT linac, and finally tomotherapy. The $\mathrm{GI}_{\text {Paddick }}$ value of the FFF-VMAT linac for large-diameter tumors $(28 \mathrm{~mm})$ was better than that of tomotherapy, with ratios of 3.67: 4.14 and 3.21: 3.90 for distances of 1 and $6 \mathrm{~mm}$ from the brainstem. However, tomotherapy had better $\mathrm{GI}_{\text {Paddick values than the FFF-VMAT linac for }}$ smaller-diameter tumors $(8 \mathrm{~mm})$, with ratios of 9.07 : 10.97 and 8.57: 10.58 for distances of 1 and $6 \mathrm{~mm}$ from the brainstem, respectively.

\section{Dose measurements}

The dose-linearity curves of TLD and EBT3 (0-800 cGy) are shown in Fig. 4. The radiation doses at the center of the tumors within the phantom were clinically measured and compared with the dose calculated in the treatment plan; the differences are shown in Table 2. The TLD and EBT3 dose measurements indicated that the average differences between the output doses of the cone-based linac, the FFF-VMAT linac, and tomotherapy and the calculated doses of the treatment plans were generally lower than $4 \%$. The largest observed difference was $-3.68 \%$ $(-4.52 \%$ to $-3.27 \%)$ for the cone-based linac and was measured using the EBT3 film.

Figure 5 shows the dose profile measured using EBT3 films for tumors locaed $1 \mathrm{~mm}$ from the brainstem in the $\mathrm{R}-\mathrm{L}$ and $\mathrm{S}-\mathrm{I}$ directions. The results of the measurement using the EBT3 film are generally consistent with calculations of the treatment plan. The average dose profile widths of the $50 \%$ and $30 \%$ doses relative to the dose at the center of the tumor for tumors of various sizes are shown in Fig. 6. The 30\% profile widths of the FFFVMAT linac were smaller than those of tomotherapy for all tumor volumes because the gantry rotated in a coplanar fashion, synchronized with the movement of the treatment couch, during the tomotherapy treatments to enable irradiation of the tumor. Because the $\mathrm{R}-\mathrm{L}$ direction lies within the beam pathway, the distribution of low-intensity doses in the $\mathrm{R}-\mathrm{L}$ direction was broadened.

The gamma passing rates of the three treatment modalities under two different gamma passing criteria are shown in Fig. 7. When the 3\% 3-mm criteria was used as the passing metric, the passing rates for the conebased linac, the FFF-VMAT linac, and tomotherapy were $99.28 \pm 1.36 \%$, $98.71 \pm 1.39 \%$, and $99.98 \pm 0.18 \%$, respectively. Among these, tomotherapy had the highest passing rate $(p<0.05)$, and there was no statistically significant difference between the passing rates for the 

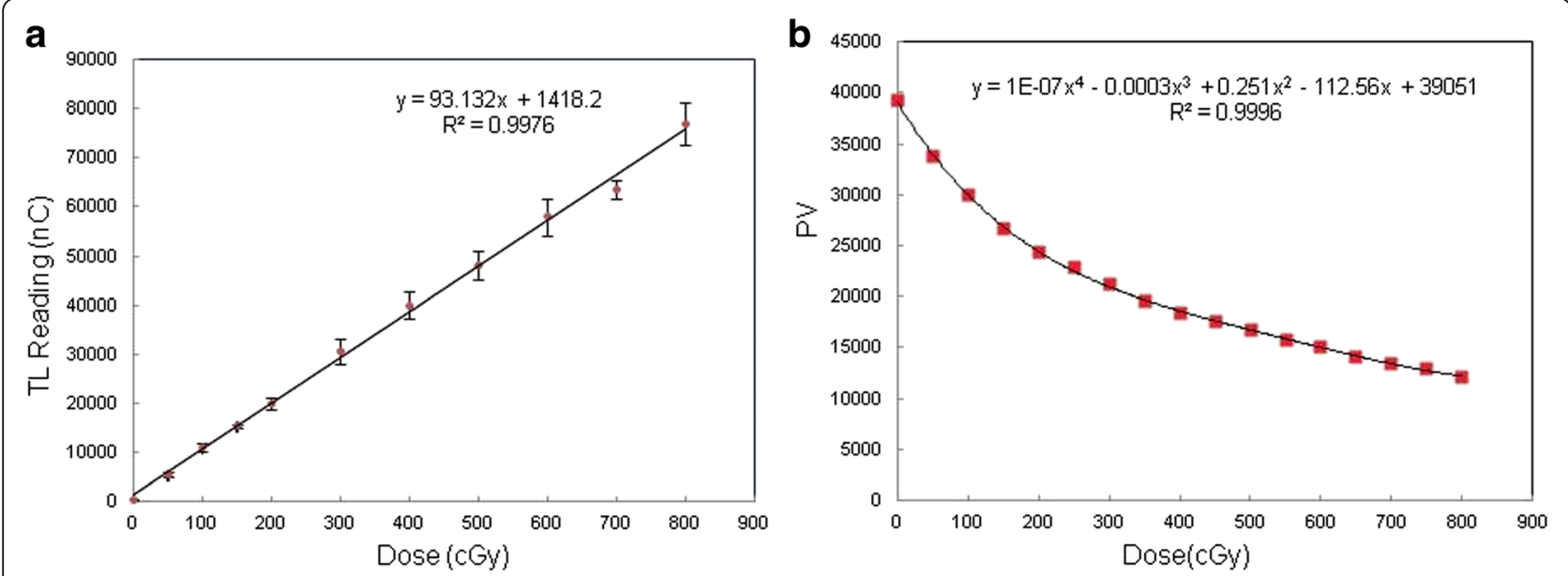

Fig. 4 Dose-linearity curves for $6 \mathrm{MV}$ : (a) TLD and (b) EBT3

cone-based and FFF-VMAT linacs $(p=0.235)$. Considering that SRT treatments characteristically require a high level of positional accuracy and steep dose gradients, the treatment modalities were also evaluated using the 5\% $1-\mathrm{mm}$ criteria as the passing metric. In this case, the passing rates for the cone-based linac, the FFF-VMAT linac, and tomotherapy were $97.73 \pm 2.42 \%, 93.53 \pm$ $3.82 \%$, and $98.19 \pm 2.09 \%$, respectively. Among these, the FFF-VMAT linac had the lowest passing rate $(p<0.01)$, and there was no significant difference between the passing rates for the cone-based linac and tomotherapy ( $p=0.46)$.

The gamma evaluation maps of $28 \mathrm{~mm}$ diameter tumors located $1 \mathrm{~mm}$ from the brainstem using the 3\% 3$\mathrm{mm}$ and 5\% 1-mm criteria were analyzed, as shown in Fig. 8. Here, changing the passing metric caused an increase in the failed areas in regions with steep doses at the borders of the tumor for the cone-based and FFFVMAT linac modalities. The cone-based linac and tomotherapy retained a gamma passing rate of $95 \%$ and above, whereas the passing rate of the FFF-VMAT linac decreased below $95 \%$.

\section{Discussion}

The result of this study show that the average $\mathrm{HI}$ for the cone-based linac was lower than those for the FFFVMAT linac and tomotherapy because of the radiation of the cone-based linac was homogeneous. As a result of no dose-intensity control within the radiation field of cone-based linac, there were no significant changes for a cone treatment at a distance difference of only $5 \mathrm{~mm}$ distance to brainstem.

Because the radiation field of the cone-based linac was similar to the size of the tumors, this modality yielded the highest CGIc and CIPaddick. Unlike the cone-based linac, tomotherapy uses a constant jaw size, leading to a "ramp-up" effect, which causes normal tissues surrounding the tumor in the S-I direction to receive a higher dose [23].

The use of a cone in the cone-based linac minimized the dose divergence, resulting in the cone-based linac having the best CGIg and GIPaddick among the three treatment modalities. When the tumor volume was very small, the jaw used in tomotherapy limited the dispersion range of the low-dose region, causing the CGIg to be higher than that for the FFF-VMAT linac. As the tumor volume increased, the dose on the brainstem increased.

The research of Yip et al. [12] indicated that, regardless of the conformity and gradient, the cone-based linac performed better than tomotherapy for tumors with a regular shape; this finding is consistent with our results. However, Soisson et al. [24] reported that excellent levels of tumor conformity were achieved using tomotherapy. Nevertheless, we obtained a similar result with respect to the overall evaluation of the CGI. Soisson et al. did not provide a detailed comparison between the doses of the FFF-VMAT linac and tomotherapy for SRT, whereas

Table 2 Average difference in the dose at the center of tumors between the calculated doses from the treatment planning system and the doses measured using TLD and EBT3 for the cone-based linac, the FFF-VMAT linac, and tomotherapy

\begin{tabular}{llll}
\hline Dosimeter & Cone-based linac & FFF-VMAT linac & Tomotherapy \\
\hline TLD (\%) & $-1.38(-4.08$ to 0.81$)$ & $-1.34(-4.96$ to 4.04$)$ & $1.05(-2.10$ to 3.65$)$ \\
EBT3 (\%) & $-3.68(-4.52$ to -3.27$)$ & $-1.75(-4.70$ to 2.52$)$ & $-1.02(-3.72$ to 0.58$)$ \\
\hline
\end{tabular}




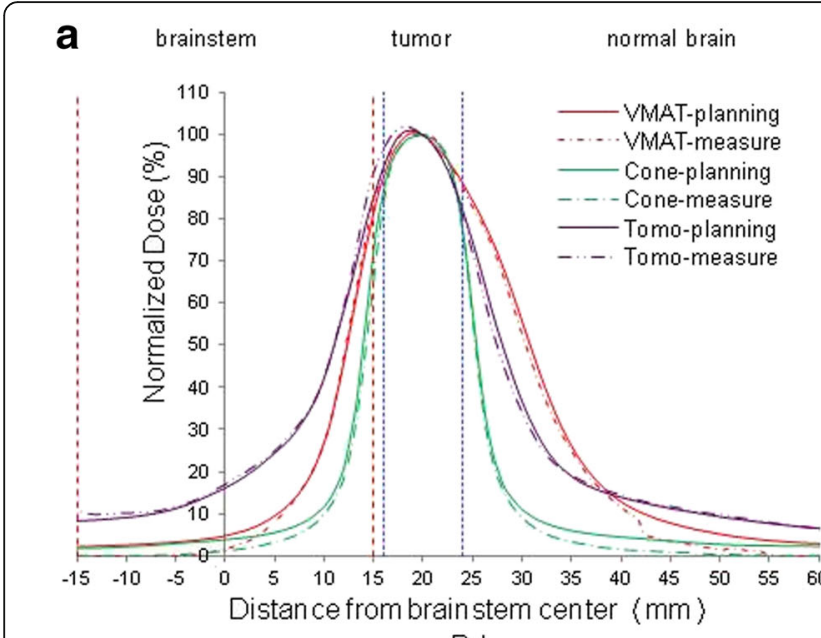

R-L

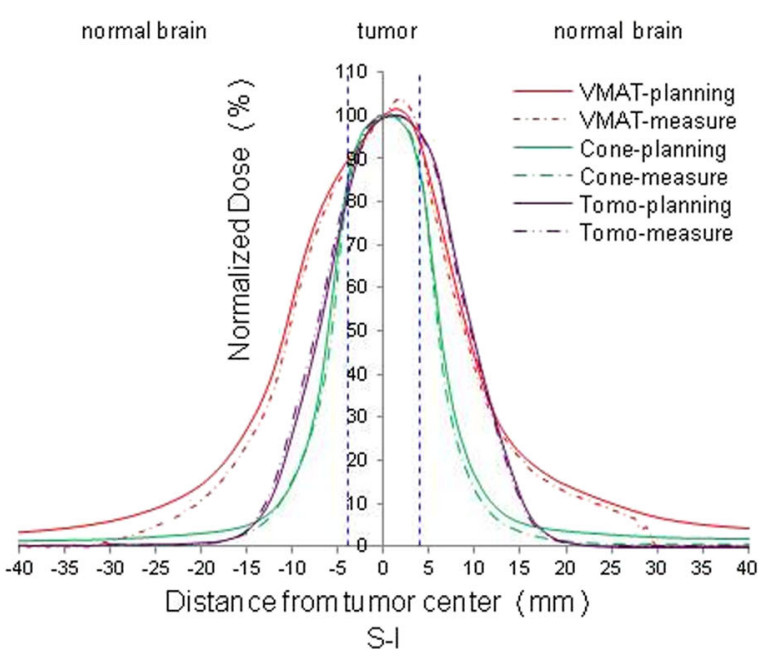

b

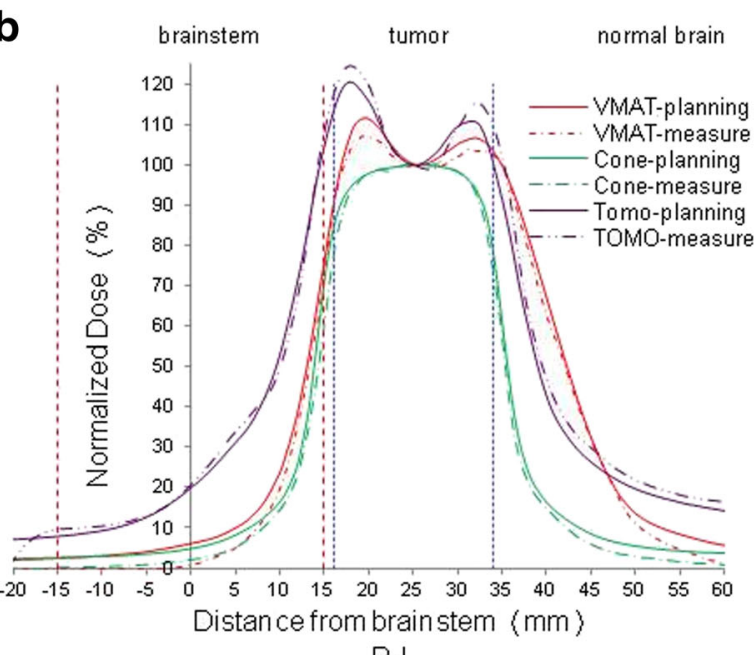

R-L

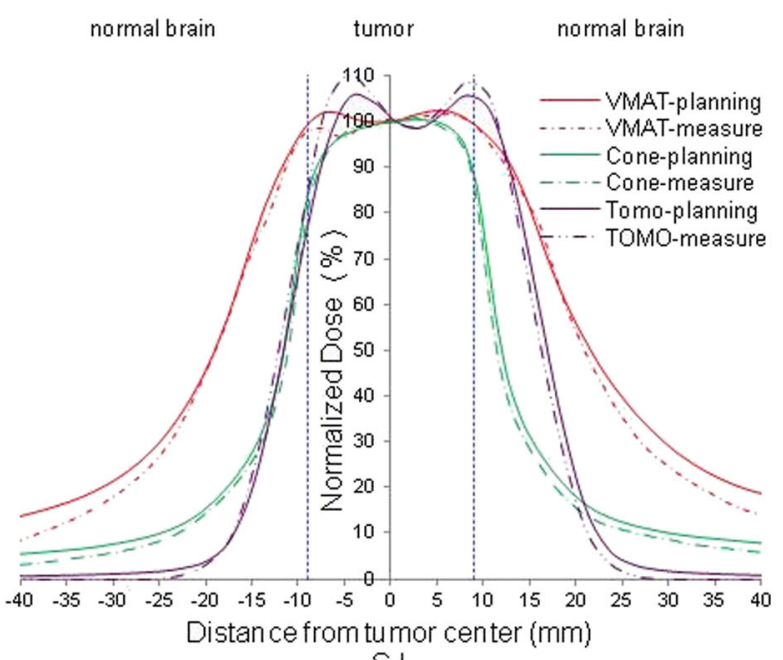

C

brainstem

tumor

normal brain

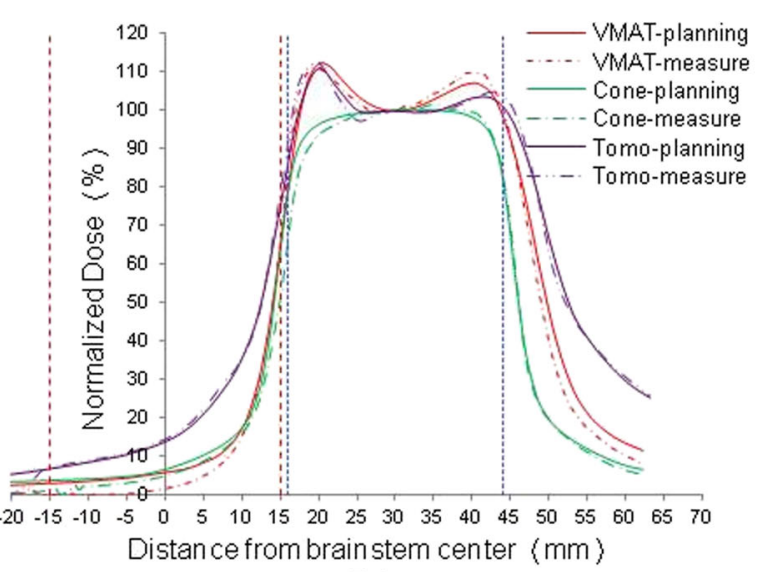

R-L

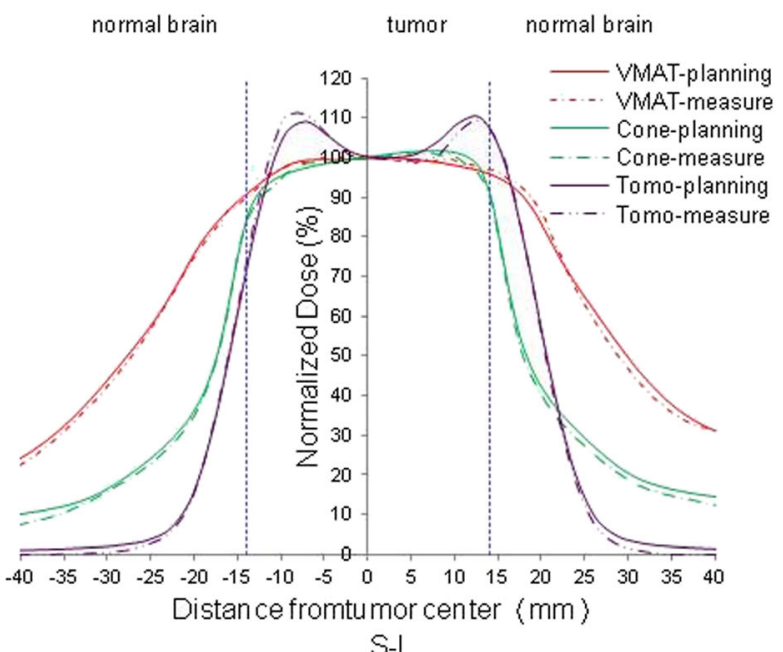

Fig. 5 Differences of EBT3-measured and treatment plan-calculated profiles (normalized to the dose of tumor center) in the R-L and $S-I$ directions for tumors located $1 \mathrm{~mm}$ from the brainstem with diameters of (a) $8 \mathrm{~mm}$, (b) $18 \mathrm{~mm}$, and (c) $28 \mathrm{~mm}$ 

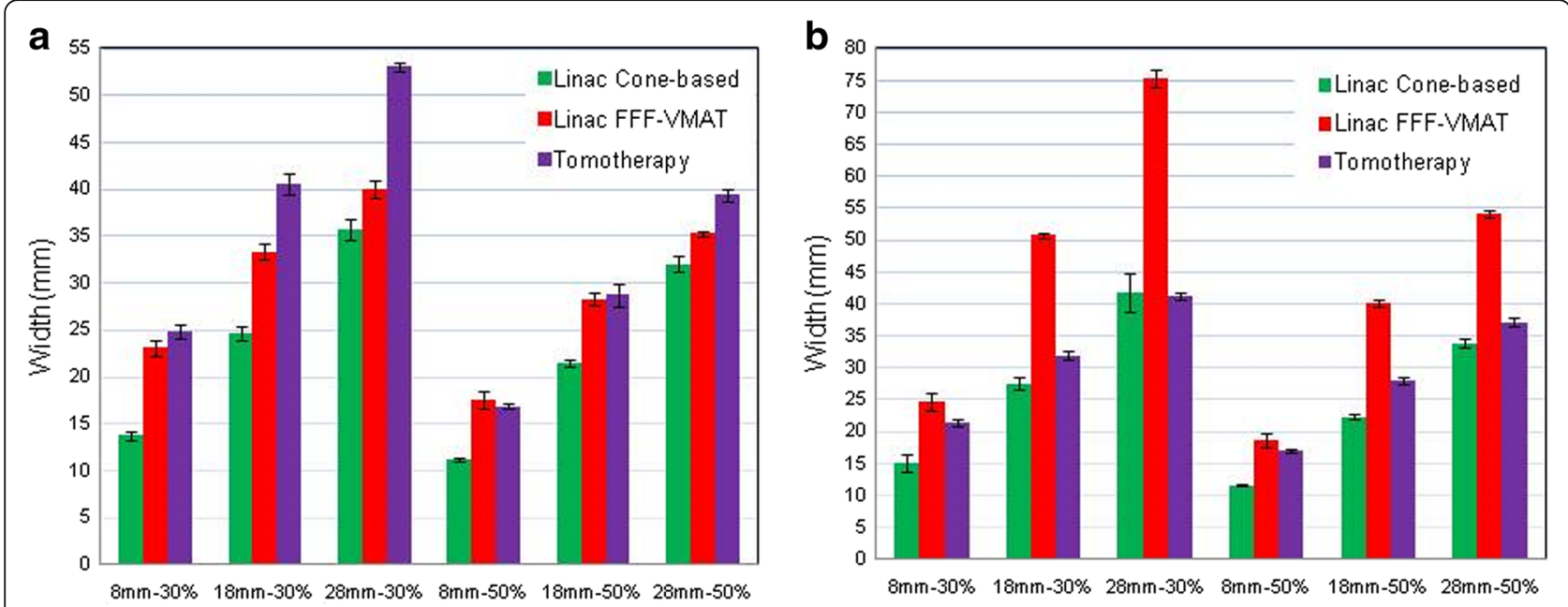

Fig. 6 Average dose profile widths of doses that are 50\% and 30\% of the dose at the center of the tumor, measured using EBT3 films, in the (a) $\mathrm{R}-\mathrm{L}$ direction and (b) S-I direction

we showed that, regardless of the tumor size and distance from the brainstem, the conformity of the FFFVMAT linac was always better than that of tomotherapy. The FFF-VMAT linac also had a steeper gradient than tomotherapy for larger tumors, whereas tomotherapy exhibited a steeper gradient for smaller tumors.

It will result in the dose accumulation to the borders of $18 \mathrm{~mm}$ and $28 \mathrm{~mm}$ diameter PTV located $1 \mathrm{~mm}$ from brainstem if we try to lower the dose of brainstem for FFF-VMAT linac and tomotherapy with intensity modulated field. In this situation, if we take into account the tumor coverage, it may increase the dose closed to the borders of PTV. For cone-based linac without intensity modulated field, the dose in the PTV will be more uniform and the dose of brainstem will be reduced because of high dose gradient. According to the AAPM TG-142 report, a deviation of $1 \mathrm{~mm}$ is allowed in the rotational center of the couch and gantry of the linear accelerator used for SRS or SRT, and the repeatability of the MLC is required [25]. These deviations could have affected the gamma analysis results for regions with a steep dose gradient. O'Connor et al. [26] reported that errors in the position of the MLC significantly affected the results of gamma analysis; for example, with the $3 \% 1-\mathrm{mm}$ criteria as the gamma analysis passing metric, a $0.8 \mathrm{~mm}$ deviation in the position of the MLC reduced the gamma passing rates of square-field rotating beams with field sizes of 16 and $40 \mathrm{~mm}$ by $5.7 \%$ and $4.5 \%$, respectively. In the continuous delivery of VMAT, the position of MLC at each control point must match the gantry's speed and dose rate, and the MLC position in this continuous process will also be affected by gravity and the speed of

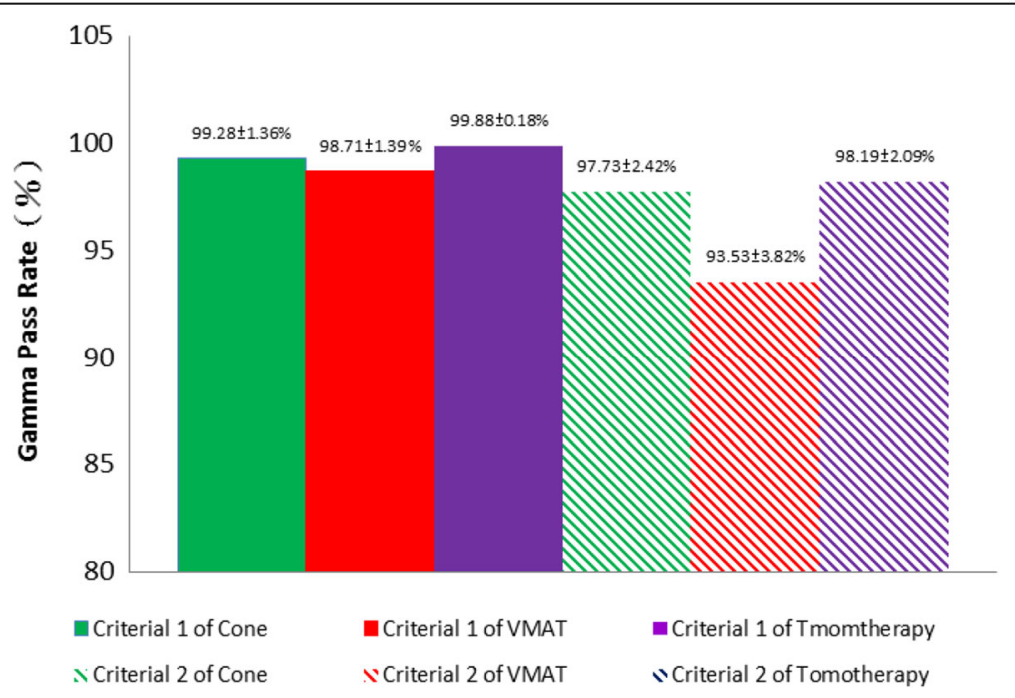

Fig. 7 Gamma passing rates of the three treatment modalities under two different gamma passing criteria: Criteria 1:3\% 3-mm and Criteria 2: 5\% 1-mm 

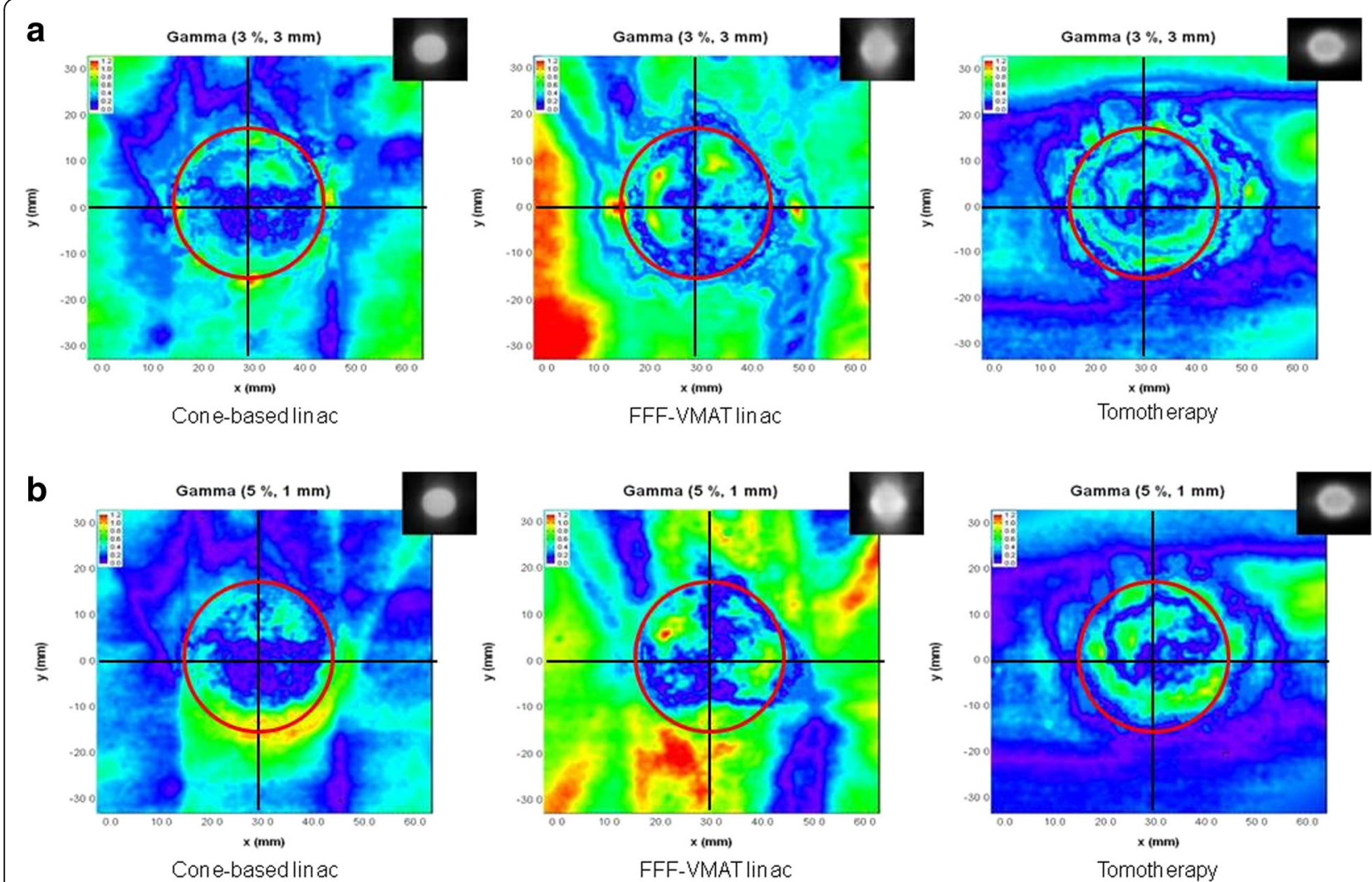

Fig. 8 Gamma-evaluation maps of the three different treatment modalities evaluated using two different gamma passing criteria (the red circle indicates the area of the $28 \mathrm{~mm}$ tumor that is located $1 \mathrm{~mm}$ from the brainstem): (a) $3 \% 3-\mathrm{mm}$ and (b) $5 \%$ 1-mm

MLC. Since the setting of MLC's tolerance table in machine's setting is $1 \mathrm{~mm}$, it means that the machine will keep delivery as the MLC position error is less than $1 \mathrm{~mm}$ at each control point in the process of delivery, and that the $1 \mathrm{~mm}$ tolerance may affect the measurement results, especially in the small field and high-dose gradient regions $[27,28]$. Therefore the more mechanical error variable, the more likely to affect the Gamma pass rate.

The treatment time of each technique is also the focus of our concern. In our study, the longest treatment time required for the cone-based linac, FFF-VMAT linac and Tomotherapy were 830, 679 and 728 s, respectively. The above time did not include the time of image registration and confirmation. For cone-based linac and FFF-VMAT linac techniques using the non-coplanar angles, the number of beams that need to rotate the angle of the couch which can affects the time required for treatment.

\section{Conclusion}

As a result of the use of spherical tumors, we could not be affected by the shape of the tumor and clearly understand the differences of the dose characteristics between three modalities. Among the three treatment modalities studied, the cone-based linac had the best conformity and dose gradient for tumors of all sizes and locations. According to our results, if a critical organ, such as the brainstem, is located near the tumor and the situation requires a steep dose gradient, the cone-based linac should be used for SRT therapy. Since the steep dose gradient of the cone-based linac is obvious, we should also consider using a high dose gradient of the cone base to treat slightly irregular tumor and protect the critical organs or normal brain. The dose conformity of the FFF-VMAT linac for tumors of all sizes and positions was better than that of tomotherapy. The dose gradient of the FFF-VMAT linac for large tumors $(28 \mathrm{~mm}$ in diameter) was better than that of tomotherapy, whereas tomotherapy had a better dose gradient than the FFFVMAT linac for small tumors $(8 \mathrm{~mm}$ in diameter). The cone-based linac had the smallest $50 \%$ and 30\% dose profile widths in the $\mathrm{R}-\mathrm{L}$ and $\mathrm{S}-\mathrm{I}$ directions among the three modalities, with the exception of the 30\% dose profile width for $28 \mathrm{~mm}$ tumors in the S-I direction, where tomotherapy and the cone-based linac produced similar results. On one hand, the $30 \%$ dose profile widths of the FFF-VMAT linac in the $\mathrm{R}-\mathrm{L}$ direction for all tumor volumes were smaller than those of tomotherapy. Therefore, for protecting normal tissues located superior and inferior to the tumor, we can consider using 
tomotherapy or Cone base with couch at $0^{\circ}$ for treatment. The TLD and EBT3 measurement results indicate that all three SRT treatment modalities achieved accurate doses. However, the FFF-VMAT and cone-based linacs may have produced dose deviations in regions with steep gradients on the borders of the tumor because of the effects of mechanical factors.

\section{Abbreviations}

3D: Three-dimensional; CGI: Conformity/gradient index; CGlc: Conformity

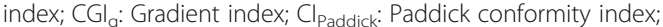
CT: Computed-tomography; FFF: Flattening filter-free; Gl paddick: Paddick gradient index; HI: Homogeneity index; Linac: Linear accelerator; MLCs: Multileaf collimators; R-L: Right-left; S-l: Superior-inferior; SRS: Stereotactic radiosurgery; SRT: Stereotactic radiotherapy; TLD: Thermoluminescent dosimeter; VMAT: Volumetric modulated arc therapy

\section{Acknowledgements}

Not applicable.

\section{Funding}

This study was supported in part by the Ministry of Science and Technology of Taiwan (MOST 104-2314-B-010-040-MY3 and MOST 106-NU-E-010-001-NU).

\section{Availability of data and materials}

All data generated or analysed during this study are included in this published article.

\section{Authors' contributions}

$\mathrm{SMH}$ and $\mathrm{YCL}$ designed the project. SMH,YCL, CCJ and $\mathrm{CYT}$ contributed to acquisition of data and data analysis. SMH,YCL and CCJ contributed to methodology of the process. SMH was the Senior Author who oversaw the project. All authors read and approved the final manuscript.

\section{Ethics approval and consent to participate}

Not applicable.

\section{Consent for publication}

Not applicable.

\section{Competing interests}

The authors declare that they have no competing interests.

\section{Publisher's Note}

Springer Nature remains neutral with regard to jurisdictional claims in published maps and institutional affiliations.

\section{Author details \\ 'Medical Physics and Radiation Measurements Laboratory, National Yang-Ming University, Taipei, Taiwan, ROC. ${ }^{2}$ Department of Biomedical Imaging and Radiological Sciences, National Yang-Ming University, No. 155, Sec. 2, Li-Nong St., Beitou District, Taipei 112, Taiwan, ROC. ${ }^{3}$ Biophotonics and Molecular Imaging Research Center, National Yang-Ming University, Taipei, Taiwan, ROC. ${ }^{4}$ Department of Physics, National Chung Hsing University, Taichung, Taiwan, ROC. ${ }^{5}$ Department of Radiation Oncology, Changhua Christian Hospital, Changhua, Taiwan, ROC.}

\section{Received: 20 April 2017 Accepted: 7 September 2017}

Published online: 16 September 2017

\section{References}

1. Lee N, Isaacson SR, Schiff PB, Sisti MB, Germano IM. Historical perspective and basic principles of radiation physics and biology. In: Germano IM, editor. LINAC and gamma knife radiosurgery. Park Ridge: AANS; 2000. p. 3-10.

2. Meeks SL, Buatti JM, Bova FJ, Friedman WA, Mendenhall WM. Treatment planning optimization for linear accelerator radiosurgery. Int J Radiat Oncol Biol Phys. 1998;41:183-97.
3. Hazard L, Wang B, Skidmore TB, Chern SS, Salter BJ, Jensen RL, et al. Conformity of linac-based stereosurgery radiosurgery using dynamic conformal arcs and micro-multileaf collimator. Int J Radiat Oncol Biol Phys. 2009;73:562-70.

4. Dhabaan A, Elder E, Schreibmann E, Crocker I, Curran WJ, Oyesiku NM, et al. Dosimetric performance of the new high-definition multileaf collimator for intracranial stereotactic radiosurgery. J Appl Clin Med Phys. 2010;11:197-211.

5. Mayo CS, Ding L, Addesa A, Kadish S, Fitzgerald TJ, Moser R. Initial experience with volumetric IMRT (rapidarc) for intracranial stereotactic radiosurgery. Int J Radiat Oncol Biol Phys. 2010;78:1457-66.

6. Ohtakara K, Hayashi S, Hoshi H. Dose gradient analyses in Linac-base intracranial stereotactic radiosurgery using Paddicks gradient index: consideration of the optimal method for plan evaluation. J Radiat Res. 2011;52:592-9.

7. Galal MM, Keogh S, Khalil S. Dosimetric and mechanical characteristics of a commercial dynamic HMLC used in SRS. Med Phys. 2011;38:4225-31.

8. Audet C, Poffenbarger BA, Chang P, Jackson PS, Lundahl RE, Ryu SI, et al. Evaluation of volumetric modulated arc therapy for cranial radiosurgery using multiple noncoplanar arcs. Med Phys. 2011:38:5863-75.

9. Hong LX, Gard M, Lasala P, Kim M, Mah D, Chen CC, et al. Experience of micromultileaf collimator linear accelerator based single fraction stereotactic radiosurgery: Tumors dose inhomogeneity, conformity, and dose fall off. Med Phys. 2011;38:1239-47.

10. Gevaert T, Levivier M, Lacornerie T, Verellen D, Engels B, Reynaert N, et al. Dosimetric comparison of different treatment modalities for stereotactic radiosurgery of arteriovenous malformations and acoustic neuromas. Radiother Oncol. 2013;106:192-7.

11. Kaul D, Badakhshi H, Gevaret T, Pasemann D, Budach V, Tuleasca C, et al. Dosimetric comparison of different treatment modalities for stereotactic radiosurgery of meningioma. Acta Neurochir. 2015;157:559-63.

12. Yip HY, Mui WL, Lee JW, Fung WW, Chan JM, Chiu G, et al. Evaluation of radiosurgery techniques-Cone-based linac radiosurgery vs tomotherapybased radiosurgery. Med Dosim. 2013;38:184-9.

13. Shaw E, Scott C, Souhami L, Dinapoli R, Bahary JP, Kline R, et al. Radiosurgery for the treatment of previously irradiated recurrent primary brain tumors and brain metastases: Initial report of Radiation Therapy Oncology Group Protocol 90-05. Int J Radiat Oncol Biol Phys. 1996;34:647-54

14. Wagner TH, Bova FJ, Friedman WA, Buatti JM, Bouchet LG, Meeks SL. A simple and reliable index for scoring rival stereotactic radiosurgery plans. Int J Radiat Oncol Biol Phys. 2003:57:1141-9.

15. Paddick I. A simple scoring ratio to index the conformity of radiosurgical treatment plans. J Neurosurg. 2000;93(Suppl 3):219-22.

16. Paddick I, Lippitz B. A simple dose gradient measurement tool to complement the conformity index. J Neurosurg. 2006;105(Suppl):194-201.

17. Ertl A, Zehetmayer M, Schöggl A, Kindl P, Hartl R. Dosimetry studies with TLDs for stereotactic radiation techniques for intraocular tumors. Phys Med Biol. 1997:42:2137-45.

18. Ho AK, Gibbs IC, Chang ST, Main B, Adler JR. The use of TLD and Gafchromic film to assure submillimeter accuracy for image-guided radiosurgery. Med Dosim. 2008:33:36-41.

19. Faught AM, Kry SF, Luo D, Molineu A, Bellezza D, Gerber RL, et al. Development of a modified head and neck quality assurance phantom for use in stereotactic radiosurgery trials. J Appl Clin Med Phys. 2013;14:206-15.

20. Fiandra C, Fusella M, Giglioli FR, Filippi AR, Mantovani C, Ricardi U, et al. Comparison of Gafchromic EBT2 and EBT3 for patient-specific quality assurance: Cranial stereotactic radiosurgery using volumetric modulated arc therapy with multiple noncoplanar arcs. Med Phys. 2013; doi: 10. $1118 / 1.4816300$

21. Barbosa NA, da Rosa LA, Batista DV, Carvalho AR. Development of a phantom for dose distribution verification in stereotactic radiosurgery. Phys Med. 2013:29:461-9.

22. Cusmano D, Fumagalli ML, Marchetti M, Fariselli L, De Martin E. Dosimetric verification of stereotactic radiotherapy dose distributions using Gafchromic EBT3. Med Dosim. 2015:40:226-31.

23. Oliver M, Ansbacher W, Beckham WA. Comparing planning time, delivery time and plan quality for IMRT, Rapid Arc and tomotherapy. J Appl Clin Med Phys. 2009;10:117-31.

24. Soisson ET, Mehta MP, Tome WA. A comparison of helical tomotherapy to circular collimator-based linear-accelerator radiosurgery for the treatment of brain metastases. Am J Clin Oncol. 2011:34:388-94.

25. Klein EE, Hanley J, Bayouth J, Yin FF, Simon W, Dresser S, et al. Task Group 142 report: Quality assurance of medical accelerators. Med Phys. 2009:36: 4197-212. 
26. O'Connor P, Seshadri V, Charles P. Detecting MLC errors in stereotactic radiotherapy plans with a liquid filled ionization chamber array. Australas Phys Eng Sci Med. 2016;39:247-52.

27. Nithiyanantham K, Mani GK, Subramani V, Mueller L, Palaniappan KK, Kataria T. Analysis of direct clinical consequences of MLC positional errors in volumetric-modulated arc therapy using 3D dosimetry system. J Appl Clin Med Phys. 2015;16:296-305.

28. Chen F, Rao M, Ye JS, Shepard DM, Cao D. Impact of leaf motion constraints on IMAT plan quality, deliver accuracy, and efficiency. Med Phys. 2011;38: 6106-18.

Submit your next manuscript to BioMed Central and we will help you at every step:

- We accept pre-submission inquiries

- Our selector tool helps you to find the most relevant journal

- We provide round the clock customer support

- Convenient online submission

- Thorough peer review

- Inclusion in PubMed and all major indexing services

- Maximum visibility for your research

Submit your manuscript at www.biomedcentral.com/submit
Biomed Central 